Sains Malaysiana 50(11)(2021): 3193-3204

http://doi.org/10.17576/jsm-2021-5011-04

\title{
Extracting Crown Morphology with a Low-Cost Mobile LiDAR Scanning System in the Natural Environment
}

(Mengekstrak Morfologi Silara dengan Sistem Pengimbasan LiDAR Mudah Alih Kos Rendah pada Alam Sekitar Semula Jadi)

\author{
KAI WANG, JUN ZHOU*, WENHAI ZHANG \& BAOHUA ZHANG
}

\begin{abstract}
To meet the demand for intelligent measurements of canopy morphological parameters, a mobile LiDAR scanning system with LiDAR and IMU as the main sensors was constructed. The system uses a LiDAR-IMU tight coupling odometry method to reconstruct a point cloud map of the area surveyed. After using the RANSAC algorithm to remove the map ground, the European clustering algorithm is used for point cloud segmentation. Finally, morphological parameters of the canopy, such as crown height, crown diameter, and crown volume, are extracted using statistical and voxel methods. To verify the algorithm, a total of 43 trees in multiple plots of the campus were tested and compared. The algorithm defined in this study was evaluated with manual measurements as reference, and the morphological parameters of the canopy obtained using the LOAM and LeGO-LOAM algorithms as the basic framework were compared. Experiments show that this method can be used to easily obtain the crown height, crown diameter, and crown volume of the area; the correlation coefficients of these parameters were 0.91, 0.87, and 0.83, respectively. Compared with the LOAM and LeGO-LOAM methods, they were increased by 0.004, 0.12, and 0.13 and 0.07, 0.15, and 0.04, respectively. The test results for this new system are positive and meet the requirements of horticulture and orchard measurements, indicating that it will have significant value as an application.
\end{abstract}

Keywords: Canopy measurement; mobile LiDAR scanning system; point cloud segmentation; tightly-coupled odometry

\section{ABSTRAK}

Bagi memenuhi permintaan untuk pengukuran parameter morfologi kanopi yang cerdas, sistem pengimbasan LiDAR mudah alih dengan LiDAR dan IMU sebagai sensor utama telah dibina. Sistem ini menggunakan kaedah odometri gandingan erat LiDAR-IMU untuk menyusun semula peta awan titik kawasan yang dikaji. Setelah menggunakan algoritma RANSAC untuk menghilangkan landasan peta, algoritma pengelompokan Eropah digunakan untuk segmentasi awan titik. Akhirnya, parameter morfologi kanopi, seperti tinggi silara diameter silara dan isi padu silara, diekstrak menggunakan kaedah statistik dan voksel. Untuk mengesahkan algoritma, sebanyak 43 pokok di beberapa petak kampus diuji dan dibandingkan. Algoritma yang ditentukan dalam kajian ini dinilai dengan pengukuran manual sebagai rujukan dan parameter morfologi kanopi yang diperoleh menggunakan algoritma LOAM dan LeGO-LOAM sebagai kerangka asas dibandingkan. Uji kaji menunjukkan bahawa kaedah ini dapat digunakan untuk mendapatkan ketinggian silara, diameter silara dan isi padu silara dengan mudah; pekali korelasi parameter ini masing-masing 0.91, 0.87 dan 0.83. Berbanding dengan kaedah LOAM dan LeGO-LOAM, mereka masing-masing dinaikkan sebanyak 0.004, 0.12 dan 0.13 dan 0.07, 0.15 dan 0.04. Hasil ujian untuk sistem baru ini adalah positif dan memenuhi syarat pengukuran hortikultur dan kebun yang menunjukkan bahawa ia akan memiliki nilai yang signifikan sebagai aplikasi.

Kata kunci: Odometri gandingan erat; pengukuran kanopi; sistem pengimbasan LiDAR mudah alih; titik segmentasi awan

\section{INTRODUCTION}

Canopy morphological parameter measurements are an important part of precision gardening (Pfeiffer et al.
2018). Canopy information can reflect the yield and growth status of fruit trees, and provide a certain level of guidance for fertilization, irrigation, pruning, and other operations (Davison et al. 2020). Consequently, 
the development of an efficient and low-cost canopy morphology measurement method has certain practical significance. The current traditional canopy measurement methods utilize manual tape measurements or projection methods (Seka et al. 2019). These tasks are timeconsuming and laborious and measurement accuracy is closely related to the worker's proficiency. In recent years, ground LiDAR has also been utilized to determine canopy measurements and has achieved a good level of accuracy, however, it is a tedious method and its high cost hinders its promotion and development (Wang et al. 2018). In recent years, with the development of LiDAR and SLAM sensor technologies, Mobile LiDAR scanning (MLS) systems have been increasingly used for industrial and agricultural environmental understanding and target detection (Escolà et al. 2016). This has created a strong basis for the use of mobile LiDAR scanning system in tree crown measurements.

The application of early mobile scanning systems in agriculture or forestry relied on vehicle-mounted LiDAR systems. Li et al. (2016) designed a method using vehicle-mounted LiDAR and vehicle speed to measure the canopy volume of a single row of trees online, as vehicle speed is a method for point_cloud matching. However, as the vehicle speed is not guaranteed to be constant, there was a high error rate. James $P$ used a mobile robot equipped with LiDAR, camera, and GPS to measure the canopy of an entire orchard and estimate the yield, but because the robot relied on GPS for its point cloud matching, if the GPS signal was lost in the orchard, the point cloud map construction would fail (Underwood et al. 2016). With the rapid development of drone technology in recent years, morphological measurements of orchards or forests with LiDAR or cameras using GPS and IMU integrated navigation technologies, have also been widely used. Sun et al. (2019) utilized a drone equipped with a multispectral camera to map orchards and corrected for the ground, then used a probability density estimation method to segment the fruit tree, and finally used the ellipse volume to simulate the crown volume. Hadas et al. (2019) utilized drones, 3D LiDAR, and GPS systems to measure the morphology of fruit trees and achieved positive results. The use of drones to measure trees can be an appropriate choice, but in the woods, drones may not be able to obtain a complete tree point cloud, complicating the method and making it more expensive to operate. With the development of 3D-SLAM technology, the MLS system based on SLAM technology has been applied in the field of forest surveying and mapping. Zhou et al. (2019) used an MLS system based on the LOAM algorithm to measure tree diameter at breast height. Pierzchała et al. (2018) used a wheeled robot with a 3D LiDAR to map a forest using graph-SLAM technology and extract the diameter at breast height, of a single tree. These methods all use the LiDAR-IMU loose coupling method for laser mapping. When the environment has fewer feature points, it is easy to cause mismatching, map confusion, and other scenes. The recently developed LiDAR-IMU tightly-coupled odometry algorithm, have greatly improved the accuracy and robustness of point cloud map construction (Ye et al. 2019). The application of this technology will enable better results for tree crown measurements.

In response to the current needs for tools to assess the morphological measurements of orchards or forest trees, this paper proposes a new measurement method that combines LiDAR and IMU. This method uses a tightlycoupled odometry and map-matching global optimization method to construct a high precision forest point cloud map, and then uses a human-computer interaction method to obtain a more compact processing area, to reduce the number of calculations and improve segmentation efficiency. Finally, the point cloud segmentation algorithm is used to extract the information for the trees to be detected in the area's measurement domain and analyze and extract its canopy morphological parameters.

\section{MATERIALS AND METHODS}

\section{EXPERIMENTAL PLATFORM}

This project has designed portable experimental equipment for data collection and processing and designed and developed a mobile endpoint cloud processing and visualization system. The system hardware includes 3D LiDAR, an inertial measurement unit, a small industrial computer, a tablet computer, and hand-held bracket for fixing the IMU and LiDAR. The hardware composition is shown in Figure 1.

The 3D LiDAR module uses RoboSense's RS-16 LiDAR. The sensor has 16 LiDAR beams for distance measurements, ranging in accuracy of $\pm 2 \mathrm{~cm}$, a vertical viewing angle of $\pm 15^{\circ}$, a measuring distance of up to $150 \mathrm{~m}$, and angular resolution from $0.9^{\circ} \sim 0.36^{\circ}$. IMU uses the xsens series Mti-300 inertial navigation unit, which outputs high-precision pitch, roll, and yaw angles at a high frequency $(400 \mathrm{~Hz})$, and has strong seismic and anti-interference performance. The GIGABYTE-GB-B XI5H-4200 micro host was used as a portable processing module. The operating system used was ubuntu16.04, with a i7-5820k processor, $32 \mathrm{G}$ memory, and $256 \mathrm{G}$ solid 
state drive. The power supply system uses a 12V $5000 \mathrm{mAh}$ lithium battery for aerial photography. A Lenovo MIX 720 tablet computer was selected as the mobile visualization terminal.

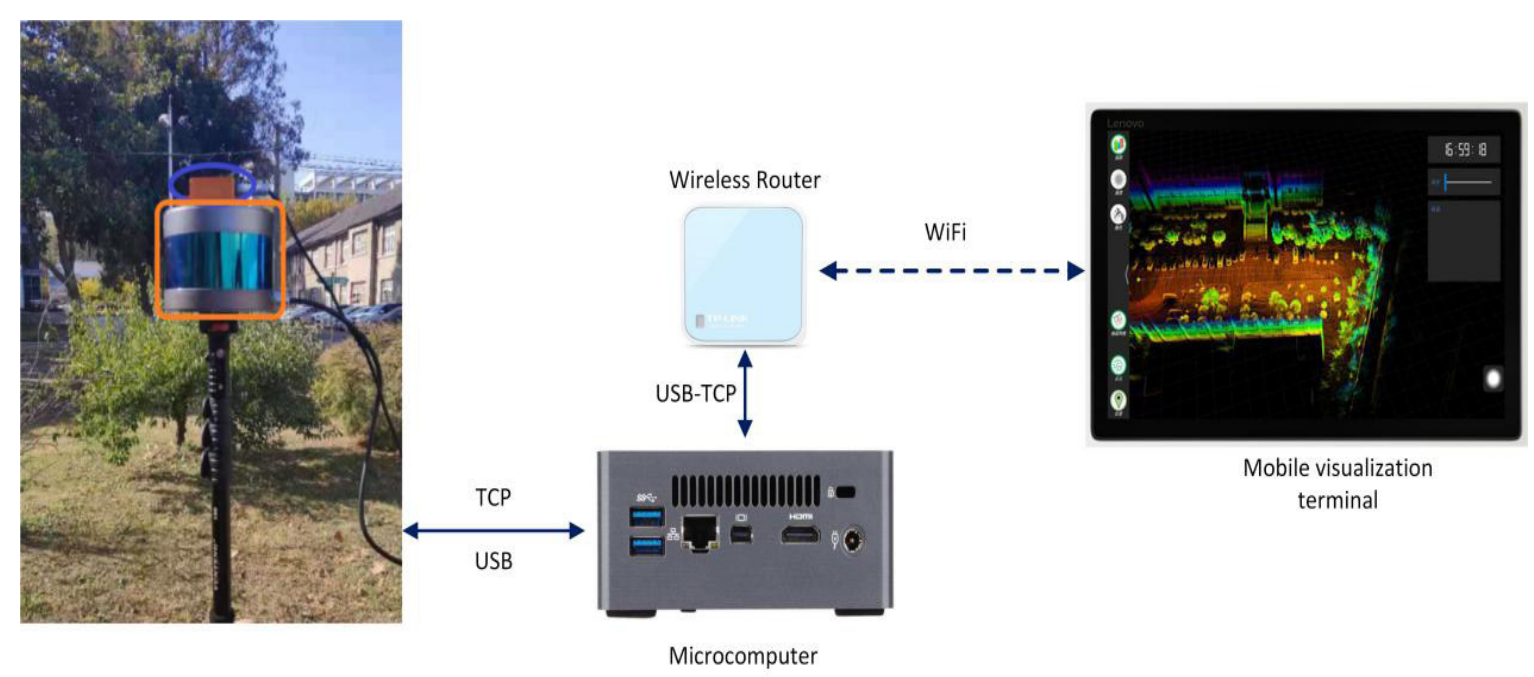

FIGURE 1. System hardware composition, the solid line is a wired connection, the dashed line is a wireless connection, the device in the orange box is lidar, and the device in the blue box is IMU

\section{SOFTWARE FRAMEWORK}

The system is mainly divided into two modules, the high-precision point cloud graph building module and the point cloud visualization and processing module. The mapping function module uses the $\mathrm{C}++$ language under the open source robot operating system (ROS) and uses the Ceres Solver library for nonlinear optimization, for optimization calculations (Rahul et al. 2020). The point cloud visualization and processing module is deployed in the windows system and uses Qt and PCL for point cloud processing and uses OpenGL3.3 (Pedersen et al. $2020)$ to realize the $3 \mathrm{D}$ point cloud visualization and other functions.

The basic process of the system is shown in Figure 2. First, it completes the real-time posture and preintegration calculation of IMU, and then uses the posture estimation of IMU to correct the motion distortion of the LiDAR and take point cloud feature points. Then, the relative measured value of the LiDAR, the pre-integration value of the IMU, and the prior term of marginalization in the sliding window, are used as parameters for jointnonlinear optimization. After obtaining the preliminary state estimation, the map matching optimization algorithm is used to obtain the accurate global state.
The original point cloud that has been converted to the world coordinate system is transmitted to the mobile processing terminal through socket communication, to establish a denser point cloud map. After obtaining all canopy information through point cloud segmentation, the morphological parameters of all canopies are extracted to fulfill the requirements of intelligent, accurate and precise gardening measurement.

\section{POINT CLOUD MAP ESTABLISHMENT}

\section{IMU dynamics}

IMU dynamics are an important part of the tightly coupled odometry algorithm. IMU global pose calculation uses the acceleration $\alpha^{\mathrm{I}}$ and angular velocity $\omega^{\mathrm{I}}$ from the accelerometer and gyroscope output in its own coordinate system I, to predict the state of IMU in the world coordinate system (Qin et al. 2018), as follows:

$$
\widetilde{\alpha}^{w}=R_{I}^{w}\left(\alpha^{I}-b_{a}^{I}-n_{a}^{I}\right)+g^{w} \quad \widetilde{\omega}^{w}=\omega^{I}-b_{\omega}^{I}-n_{\omega}^{I}
$$

where $\mathrm{g}^{\mathrm{w}}$ is the gravity vector; bI $\alpha, \mathrm{nI} \alpha$ and bI $\omega$; nI $\omega$ are the offset and noise of acceleration and angular velocity, respectively, and RW I is the rotation matrix from its own coordinate system to the world coordinate system. 


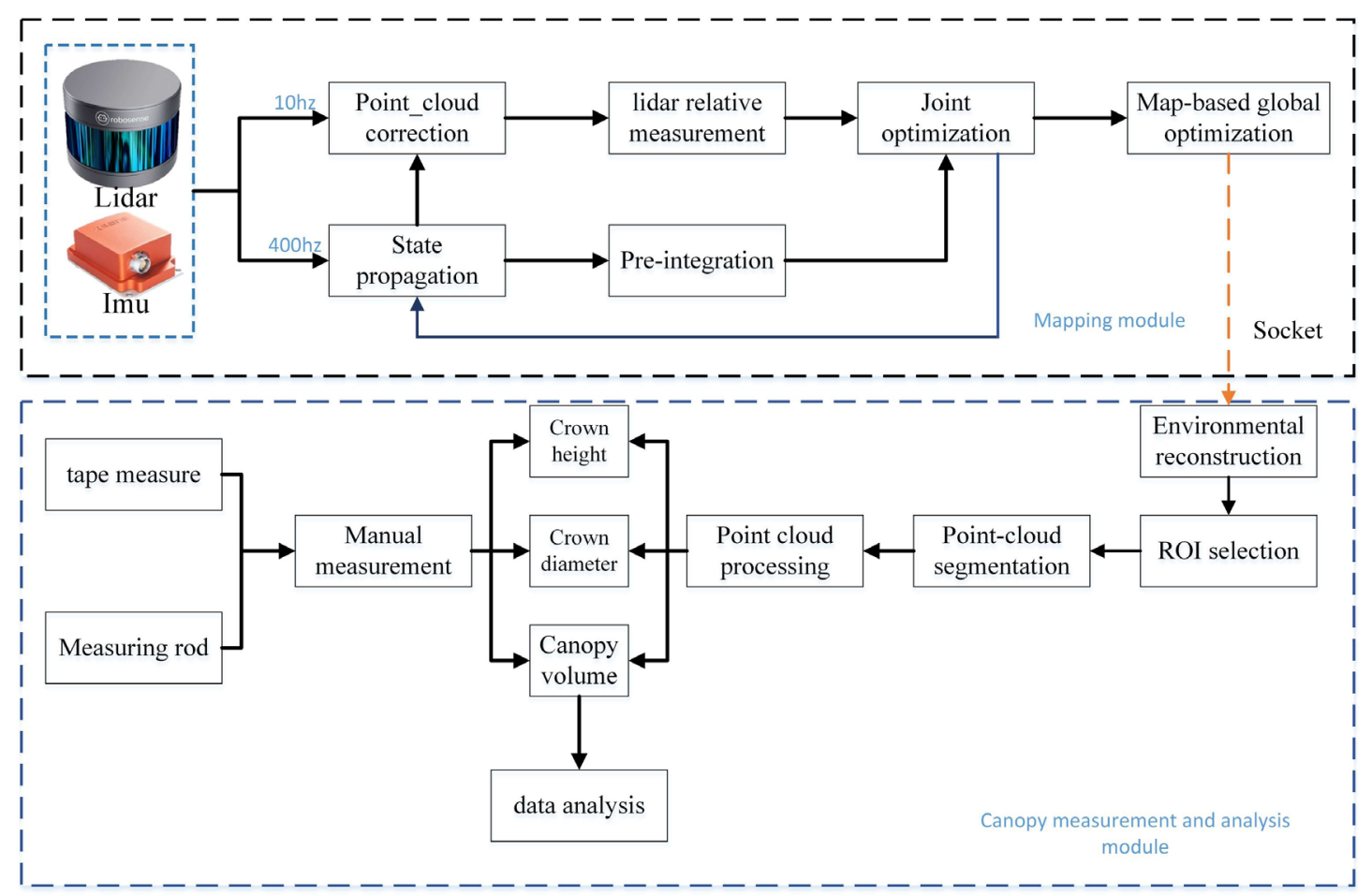

FIGURE 2. System software framework, the upper part of Figure 2 is the mapping module, and the lower part is the measurement module

After obtaining the status of IMU from the global coordinates, the real-time velocity $\mathrm{v}$, displacement $\mathrm{p}$, and direction q of IMU were calculated as follows:

$$
\begin{gathered}
v_{j}=v_{i}+\widetilde{a}_{i}^{w} \Delta t \\
p_{j}=p_{i}+v_{i} \Delta t+\frac{1}{2} \Delta t^{2}\left(\widetilde{\alpha}_{i}^{w}\right) \\
q_{j}=q_{i} \otimes\left[\begin{array}{c}
\frac{1}{2} \Delta t\left(\widetilde{\omega}^{w}\right) \\
1
\end{array}\right]
\end{gathered}
$$

where $\Delta t$ is time interval between two IMU measurements; $\mathrm{i}$ and $\mathrm{j}$ are adjacent measurement value timestamp.

Pre-integration was to integrate the IMU measurement value corresponding to the LiDAR operating frequency into a constraint of relative motion, to avoid repeated integration operations and fusion of the LiDAR and IMU in the nonlinear optimization process, pre-integration was used to describe the body motion in any time period $\mathrm{i}$ and $\mathrm{j}$ of the IMU. The pre-integration calculation formula was as follows (Qin et al. 2018):

$$
\begin{gathered}
\Delta q_{i j}=q_{i}^{T} q_{j} \\
\Delta v_{i j}=q_{i}^{T}\left(v_{j}-v_{i}-g^{w} \Delta t_{i j}\right) \\
\Delta p_{i j}=q_{i}^{T}\left(p_{j}-p_{i}-v_{i} \Delta t_{i j}-\frac{1}{2} g t_{i j}^{2}\right)
\end{gathered}
$$

Point cloud motion correction and feature-point extraction

Since the sensor is in motion during the data collection process, point cloud motion distortion will inevitably occur. To correct motion distortion, perform the following operations:

First, assume that the LiDAR motion model is a uniform motion model, and then solve the pose of the IMU in the world coordinate system, and finally use the pose of IMU to linearly interpolate the LiDAR posture to obtain the point cloud posture at any time. The point-cloud pose at any time $\mathrm{k}$ is as follows:

$$
T_{(k)}^{L}=\frac{t_{k}-t_{i}}{\Delta t} T_{(i, j)}^{L}
$$


$T^{L}(i, j)$ is the conversion matrix and $\Delta t$ is the time that lidar receives a complete point cloud, $i$ and $j$ are, respectively, the time corresponding to the start and end of LiDAR receiving a frame of point cloud.

There are ICP and feature descriptor-based matching strategies between point clouds (Segal et al. 2009), but their calculations are too large and not suitable for a real-time matching operation. According to LOAM (Zhang \& Singh 2016), the feature-points are extracted according to the calculated point cloud curvature value for subsequent matching, which means that not only are the calculation dimensions reduced but the matching efficiency improved. The point cloud curvature calculation formula is:

$$
c=\frac{1}{|S| \bullet\left\|X_{(k, j)}^{L}\right\|}\left\|\sum_{j \in S, j \neq i}\left(X_{(k, i)}^{L}-X_{(k, j)}^{L}\right)\right\|
$$

where $L$ represents the laser coordinate system; $X^{L}(k, j)$ is the $i$-th point of the LiDAR point cloud at time $k$, $j$ represents the neighboring points around $i$, and the selected interval is 5 points before and after point $i$, is the surrounding point_cloud set.

\section{Tightly coupled odometry}

Tightly-coupled odometry optimizes a variety of parameters to construct a joint loss function, with the help of nonlinear optimization methods, it iterates to minimize the errors to obtain a stable state estimation process. Tightly-coupled odometry needs to initialize the system before data processing, to obtain important parameters for the system, including the relative conversion relationship between IMU and LiDAR ( $T L B)$, the noise and bias of IMU, and the direction of gravity. According to previous research (Qin et al. 2020), system initialization can be completed through online calibration.

In order to improve the robustness of the feature matching and reduce the number of calculations, a group of adjacent point cloud was used to form a sliding window (Ye et al. 2019), as shown in Figure 3.

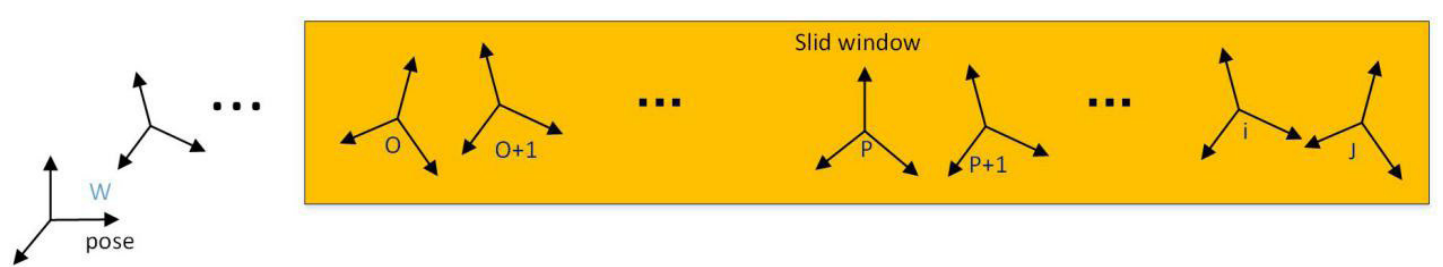

FIGURE 3. Sliding window composition, the window uses $\mathrm{p}_{\mathrm{p}}$ as the pivot point, $\mathrm{p}_{\mathrm{o}}$ as the initial point, and $\mathrm{p}_{\mathrm{j}}$ as the latest point

To optimize the LiDAR's pose from $\mathrm{P}_{\mathrm{p}+1}$ to $\mathrm{P}_{\mathrm{j}}$ in the sliding window, constrain the LiDAR and perform iterative optimizations in conjunction with the IMU, and calculate the relative measurement values of the LiDAR's pose to be optimized in the sliding window, the following calculation steps were conducted: First, build a local map in the sliding window form the $\mathrm{P}_{\mathrm{o}}$ to $\mathrm{P}_{\mathrm{i}}$ with the transformation matrix $\mathrm{T}^{\mathrm{w}}$, which is retrieved from the IMU. Second, use the K-Nearest Neighbor algorithm to search for the closest point on the local map to the original feature-point (plane feature) from $\mathrm{P}_{\mathrm{p}+1}$ to $\mathrm{P}_{\mathrm{j}}$ in the sliding window. Third, fit the searched feature-points to a plane, construct the original feature point-to-plane distance constraint equation to extract the relative measurement $(\mathrm{m})$, which includes the plane normal vector $\omega$, the distance (d) from the window origin $\left(\mathrm{P}_{\mathrm{p}}\right)$, and the original feature point $(\mathrm{x})$.
The relative measurement value $(\mathrm{m})$ was combined with LiDAR's pose to get:

$$
r_{b}=\omega^{T}\left(R_{\alpha}^{p}+P_{\alpha}^{p}\right)+d, \quad \alpha \in\{p+1, \ldots j\}
$$

Among them, $\mathrm{R}$ and $\mathrm{P}$, are rotation and translation matrices, and $r_{b}$ is the residual value to be optimized.

Marginalization is a process of decomposing the joint probability distribution into the marginal probability distribution and the conditional probability distribution, so that the older states in the sliding window can be marginalized out of the sliding window, and at the same time retained as a priori for subsequent processing. The constrained residual obtained by the pre-integration of IMU $\left(r_{c}\right)$, the residual of the relative measurement of the $\operatorname{LiDAR}\left(r_{b}\right)$ and the previous state of marginalization $\left(r_{a}\right)$, form a multivariable nonlinear least square loss function: 


$$
\min _{x} \frac{1}{2}\left\{\left\|r_{a}(X)\right\|^{2}+\sum\left\|r_{b}(X)\right\|_{c_{b}}^{2}+\sum\left\|r_{c}(X)\right\|_{c_{c}}^{2}\right\} X=\left[X_{p}^{w}, \ldots X_{j}^{w}\right]
$$

where $X$ is the LiDAR status to be optimized in the sliding window; and $c_{b}, c_{c}$, are the covariance of them.

The Ceres Solver library (Agarwal et al. 2018) was used to build a Gauss-Newton method for optimization to obtain the maximum map estimate, and the IMU posture is reversely constrained by the calculated odometry state, to suppress its drift.

\section{Map-based global optimization}

Since the output of the odometer in the previous stage is a rough point_cloud attitude, the drift of the pose will become larger and larger without processing. In order to obtain an accurate global pose, the map-based global optimization method is used to further optimize the data (Shan \& Englot 2018).

The odometry can be considered accurate at the initial moment in a short time. Therefore, the corrected point cloud at the initial moment is converted into a global map $(\bar{Q})$ according to the odometry attitude. The subsequent point_cloud $(Q)$ is matched and optimized based on $\bar{Q}$ and registered in the global map. The method was as follows. First, store the overlapping parts of $\bar{Q}$ and $Q$ in different kd-trees, and the intersecting parts are called $\bar{q}$ and $q$, respectively. Second, the feature point in $q$ searches for the closest point in $\bar{q}$ in the specified area, and performs a covariance calculation on the searched feature-points, to obtain the direction of the point set and then solve the distance between the feature-point and the point set. Third, a corresponding loss function is constructed (as follows), according to the calculated distance and using the Levenberg-Marquardt algorithm to solve the nonlinear problem.

$$
f\left(X, T^{w}\right)=d
$$

where $f$ is loss function; $d$ is the distance from the feature point to the corresponding point set of the map; $X$ and $T^{w}$ is features point and global pose to be optimized.

Set the point cloud within $60 \mathrm{~m}$ around the odometry as the optimized area, and the search range of the feature-point is a cube with the peripheral size of the feature point of $0.1 \mathrm{~m}$. The working frequency of the tightly coupled odometry is $10 \mathrm{~Hz}$, and the working frequency of the mapping algorithm is $1 \mathrm{~Hz}$. The odometry module performs global optimization every 10 frames to correct the point cloud output and registers the final optimized point cloud in the point cloud map.

\section{POINT CLOUD VISUALIZATION}

To visualize the point cloud effect on the field side and facilitate real-time data analysis, the $\mathrm{C} / \mathrm{S}$ mode was used to establish a point cloud transmission framework, and the micro-industrial computer section and the mobile terminal act, as the client and server, respectively. Since the mapping module generally uses a filtering algorithm to down-sample the generated global map, the accuracy of the plant canopy information is insufficient, so the client node encapsulates the original point cloud after the final pose optimization in the json format. The data is transmitted to the server through a tcp/socket, the server parses the data and uses the drawing function provided by the OpenGL library to perform a $3 \mathrm{D}$ reconstruction. To facilitate processing of the point cloud, extract data processing area through human-machine interactions.
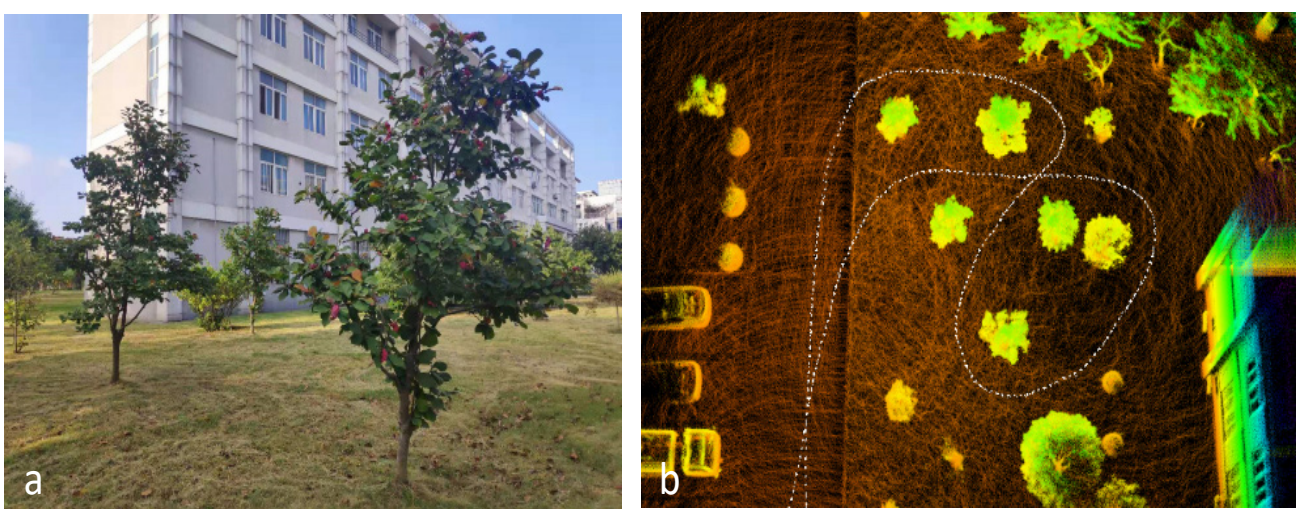

FIGURE 4. Natural environment photos and point cloud maps (a) real environment photos, and (b) reconstructed point cloud map 


\section{POINT CLOUD SEGMENTATION}

Point cloud segmentation is the complete extraction of trees from the data processing area through filtering, ground separation, and point cloud clustering algorithms. First, to remove map noise, a Statistical Outlier Removal (SOR) filter (Rusu \& Cousins 2011) was used to remove outliers based on statistical analysis of the point cloud. The SOR filtering algorithm is a statistical distribution of distances from a point to its neighbors, and for each point, the average distance from it to all its neighbors is calculated. The corresponding Gaussian distribution is obtained from the distance values, and the mean and standard deviation of the Gaussian distribution are used to determine the filtering interval, as shown in the following equation:

$$
\mu+s t d_{-} m u l * \sigma
$$

Among them, std_mul is the multiple of the standard deviation, $\mu$ and $\sigma$ are the mean and standard deviation about the desired Gaussian distribution.

In the real environment, the ground point will interfere with the system's segmentation function of the target point cloud. This paper uses the RANSAC algorithm (Qian \& Ye 2014) to classify ground and non-ground point cloud. The algorithm uses noise-containing observation data to estimate the plane mathematical equations and classify ground points through an iterative parameter method. After the ground separation is completed, the point cloud without ground information is segmented and clustered, to extract the point cloud information of each individual tree. Then the Euclidean clustering algorithm is used for point cloud clustering segmentation. The Euclidean clustering algorithm is a segmentation algorithm based on the distance between point clouds. It relies on the kd-tree for point cloud searches and uses the Euclidean distance between points as the basis for clustering. The process of the Euclidean clustering segmentation algorithm is as follows: First, find the point $\mathrm{p}_{\mathrm{i}}$ in the point cloud, search for the nearest point to $\mathrm{p}_{\mathrm{i}}$ by the kd-tree and judge the distance between the surrounding points and $\mathrm{p}_{\mathrm{i}}$. Store the points that are smaller than the threshold $\mathrm{r}$ into the sequence $\mathrm{C}$. Second, find another point $\mathrm{p}_{\mathrm{j}}$ in $\mathrm{C}$ and perform step 1 operations to update $\mathrm{C}$. Third, if the point in $\mathrm{C}$ is not updated, clear $\mathrm{C}$ and mark the point in $\mathrm{C}$ as the same type of object. Fourth, find a point outside $\mathrm{C}$ to perform the above operations, until no new points are updated, complete the segmentation of all point clouds.

Set the distance threshold of $r$ to $20 \mathrm{~mm}$ for the iterative calculation, to complete the clustering. To distinguish from weeds or other objects, the method of counting the number of point clouds is used to eliminate the point cloud blocks where the number of point clouds is less than a certain threshold (Figure 5).

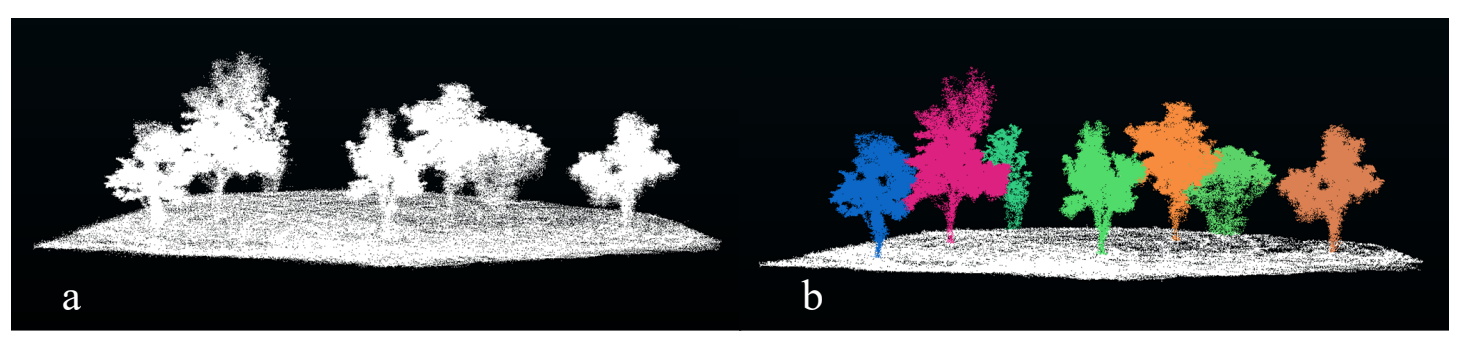

FIGURE 5. Point cloud segmentation process, (a) is the original point_cloud, (b) is the point_cloud after segmentation

\section{EXTRACTION OF CANOPY MORPHOLOGICAL PARAMETERS}

Crown morphological parameters include crown height, crown diameter, and crown volume. The parameters extracted in this paper can reflect the growth status of trees and be used as important indicators when evaluating the yield of fruit trees.

\section{Crown height and crown diameter calculations}

The first task before the canopy measurement is to determine the specific position of the canopy, that is, to find the location of the first branch point of the trunk. The specific steps are as follows: 1. the segmented tree point cloud is projected into slices from bottom to top based on the X-Y plane, and the spacing of the slice projections is $1 \mathrm{~cm}, 2$. the projection area $\mathrm{S}$ acquired for the first time is used as a reference to compare with the newly acquired projection area $\mathrm{Si}$, and 3 . when $\mathrm{Si}>1.5 * \mathrm{~S}$, the slice is regarded as the position of the first branch point, and the part above the first branch point is taken as the canopy. 
After obtaining the canopy position, the smallest external envelope box of the crown point cloud was extracted, and the crown height and crown diameter of the canopy are extracted according to the shape parameters of the envelope box.

\section{Canopy volume calculation}

The internal structure of the tree canopy is dense and complex. LiDAR uses a laser beam to obtain the complex structure of the tree canopy surface and then calculate the canopy volume. Generally, the canopy volume cannot be calculated by simply counting the number and density of points in the point cloud. The method for obtaining the canopy volume through the crown point- cloud can be divided into the convex hull method (Dong et al. 2018), Cubic Lattice Method (Fan et al. 2015), and voxel method (Langning \& Xiaoli 2019) amongst others. The convex hull method first triangulates the point cloud to close the surface, and then uses Monte Carlo and other algorithms to determine the volume. This method is simple and efficient, but due to the complexities in crown shape, it is impossible to accurately envelop the crown, and this leads to errors. The slicing method is mainly used to slice the plants from high to low, and calculate the volume of each part, according to the formula, and then the results are combined. The slicing method is suitable for cone-shaped or truncated crowns. It has no effect on other types of crown volume measurement.

Since the laser can obtain the internal structure of the crown through lidar point cloud, so this method can be used to calculate the real canopy volume. The voxel method mainly relies on the filtering algorithm to transform the point cloud into a three-dimensional raster. After the filtering is completed, the voxelized point cloud is accumulated to approximate the crown volume. This article thus uses the voxel method to complete the calculation of the tree canopy volume.

The number of voxelized point clouds is multiplied by the volume of the three-dimensional grid to determine the volume of the entire canopy. The process can be expressed as:

$$
V_{\text {voxel }}=\sum_{i=1}^{j} v
$$
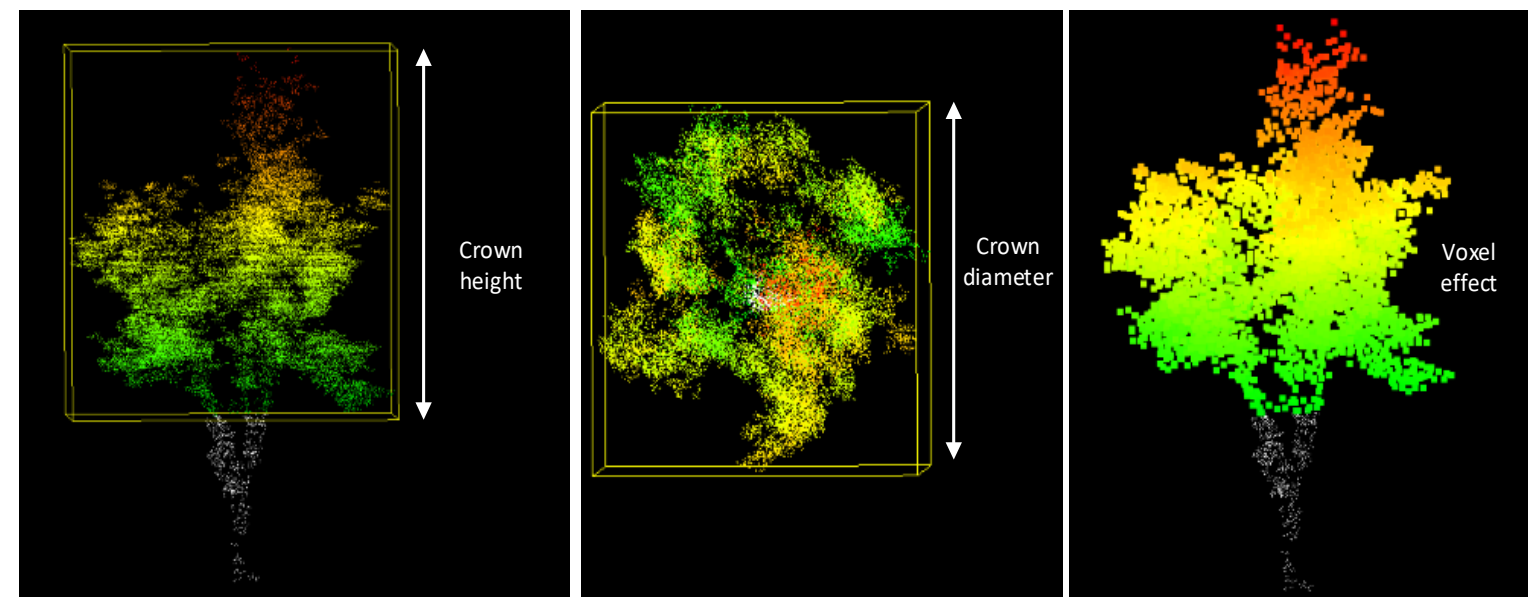

FIGURE 6. Effect of morphological feature extraction, from left to right are the effects of crown height, crown diameter extraction, and voxelized point clouds

with $V$ represents the canopy volume; $v$ represents the $3 \mathrm{D}$ grid volume; and $j$ is the number of point clouds after filtering.

\section{TEST AND ANALYSIS}

The mobile scanning devices were taken to the woods at the Pukou campus of Nanjing Agricultural University to conduct test experiments. The distance between the device and the ground was $\sim 2.2 \mathrm{~m}$, and the moving speed was controlled at $\sim 0.5 \mathrm{~m} / \mathrm{s}$. The moving track is shown in Figure 4(b), as it was a requirement that the laser beam could cover all angles of the tree to be measured. 
To obtain more objective and truer canopy information, skilled agronomists were hired to manually measure the canopy parameters. Compared with the crown diameter and crown height, the measurement of the crown volume was more difficult. The choice of grid size has a strong impact on the calculation of the crown volume. If it is too large or too small, it will affect the accuracy of the crown volume. Therefore, to obtain a better measurement value, the corresponding relationship between the two must be determined through

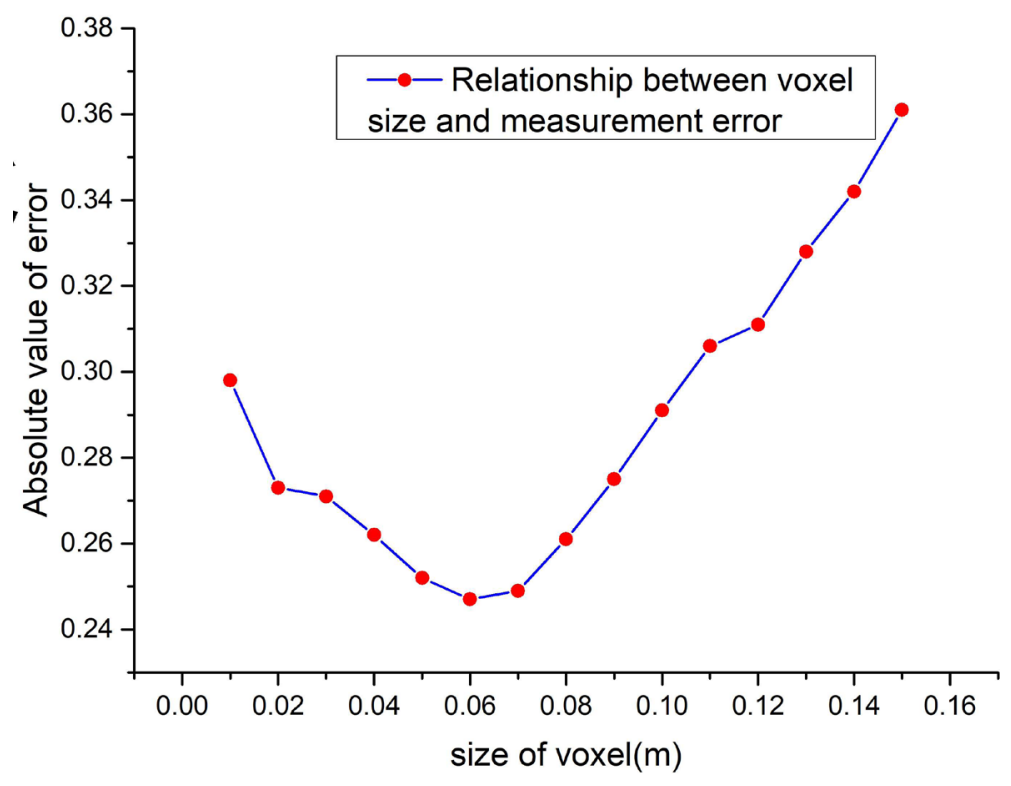

FIGURE 7. Relation between grid size and error

experiments. The canopy volume, calculated in the range of $0.01 \sim 0.15 \mathrm{~m}$, was thus compared with the manually measured value (Figure 7).

Figure 7 shows that when the voxel size is $0.06 \mathrm{~m}$, the minimum measurement error is $23.4 \%$, and the relative average error increased by $10.74 \%$, so $0.06 \mathrm{~m}$ was selected as the voxel size.

To compare the performance of the method presented in this article with other popular LiDAR odometry algorithms for tree canopy reconstruction, the latest version of the LOAM and LeGO-LOAM (Shan \& Englot 2018) algorithms were used for the comparison experiments. During the experiment, the point cloud and IMU data packets recorded by the data recording tool that was provided by the ROS, were used to reproduce the same data with the different algorithms, by playing the data packets multiple times.

The results presented in Figure 8 clearly show that the tree point clouds obtained using the algorithm presented in this paper were more detailed and similar to the real tree photos than the results from the LOAM
(Zhang \& Singh 2014) and LeGO-LOAM (Shan \& Englot 2018) algorithms. The tree canopy acceleration was determined using several algorithms for extracting test objects, and using the correlation coefficient $\mathrm{R}^{2}$ as the evaluation standard for comparison (Figure 9).

The correlation coefficient $\mathrm{R}^{2}$ obtained from this paper method was 0.759 , which is 0.004 and 0.002 higher than the canopy volume obtained using theLeGO-LOAM and LOAM methods, respectively. It is difficult to manually measure the crown volume, and consequently there are larger measurement errors. To further verify the accuracy of the reconstruction of the new crown, the relatively easy and accurate crown height and diameter from the manual measurements were used for the comparison (Figure 10).

Figure 10 shows that both crown height and crown diameter measurements have good correlation coefficients. Among them, the correlation coefficient $\mathrm{R}^{2}$ for the crown diameter, obtained from the method of this article was 0.972 , which is 0.042 and 0.09 higher than the LOAM and LeGO-LOAM methods, respectively. 
The highest measurement correlation coefficient $\mathrm{R}^{2}$ was 0.952 , which was 0.022 and 0.051 higher than that of the LeGO-LOAMand LOAM, respectively. In summary, using the tightly coupled mileage calculation method as the basis of the canopy measurement method results in more realistic tree canopy information, and the effect is better than the loosely coupled laser odometer method. However, due to the large error in the manual measurements of the crown volume itself, this correlation coefficient is lower
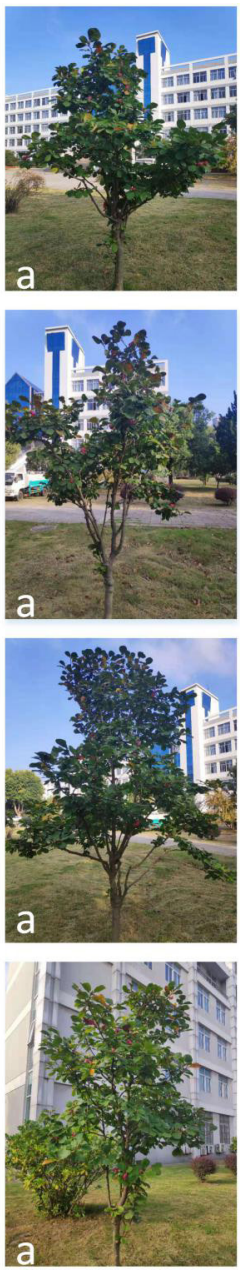
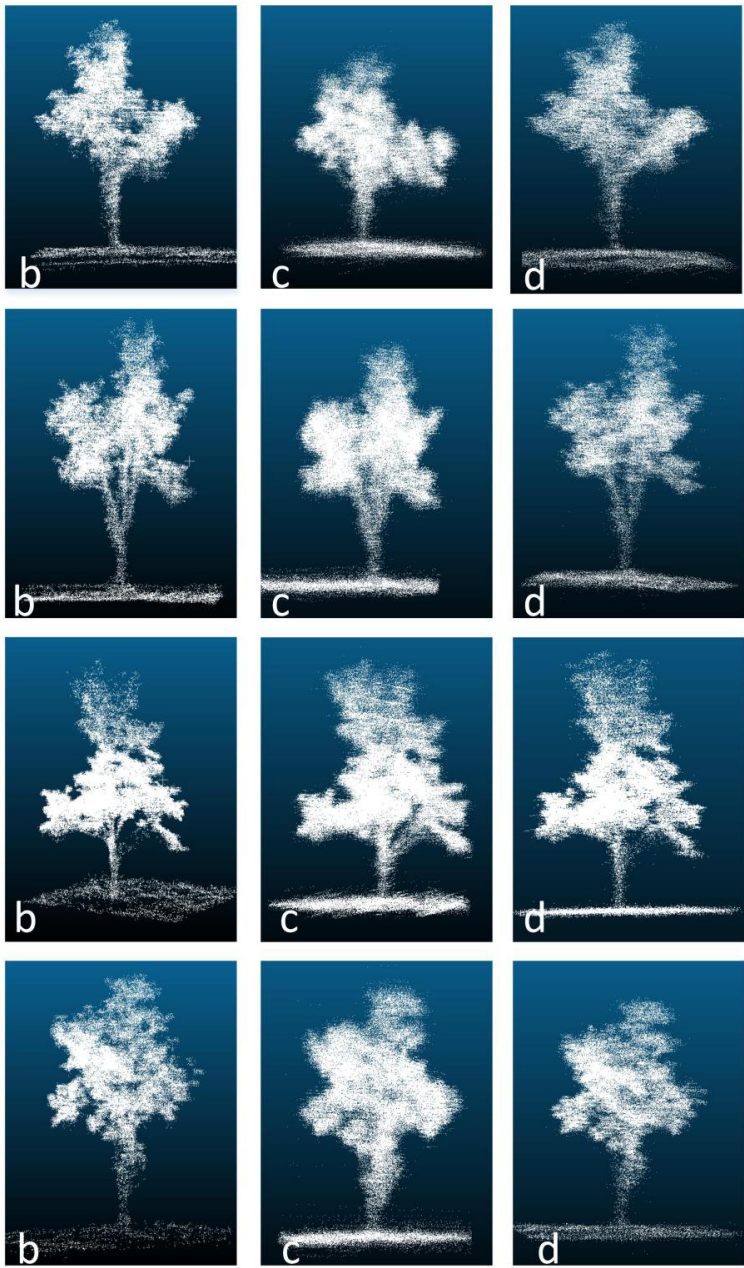
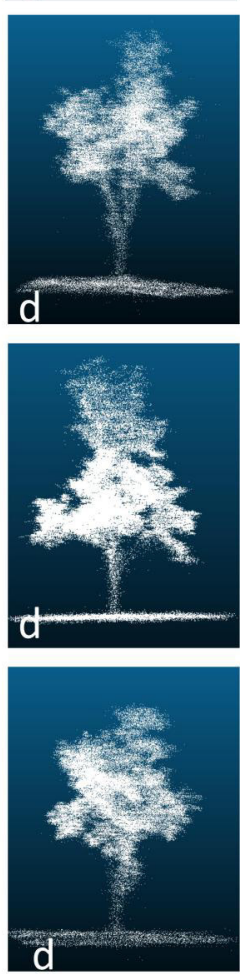

FIGURE 8. Comparison of several algorithms for point cloud reconstruction, (a) is the real photo of the tree, (b) is the final point cloud extraction effect for this article, (c) is the processing effect of loam algorithm, (d) is the processing effect of lego_loam algorithm
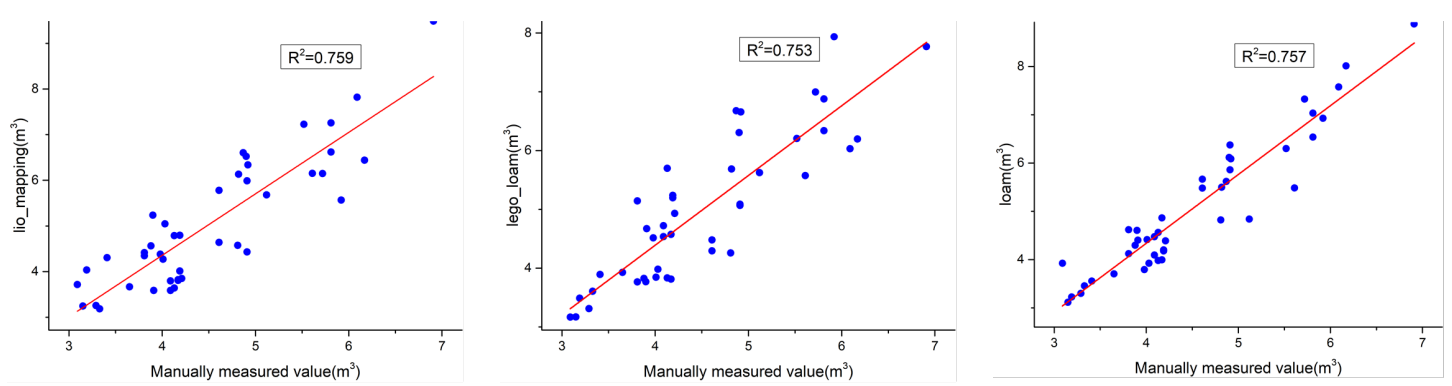

FIGURE 9. Correlation coefficient of canopy volume measurement under different algorithms 

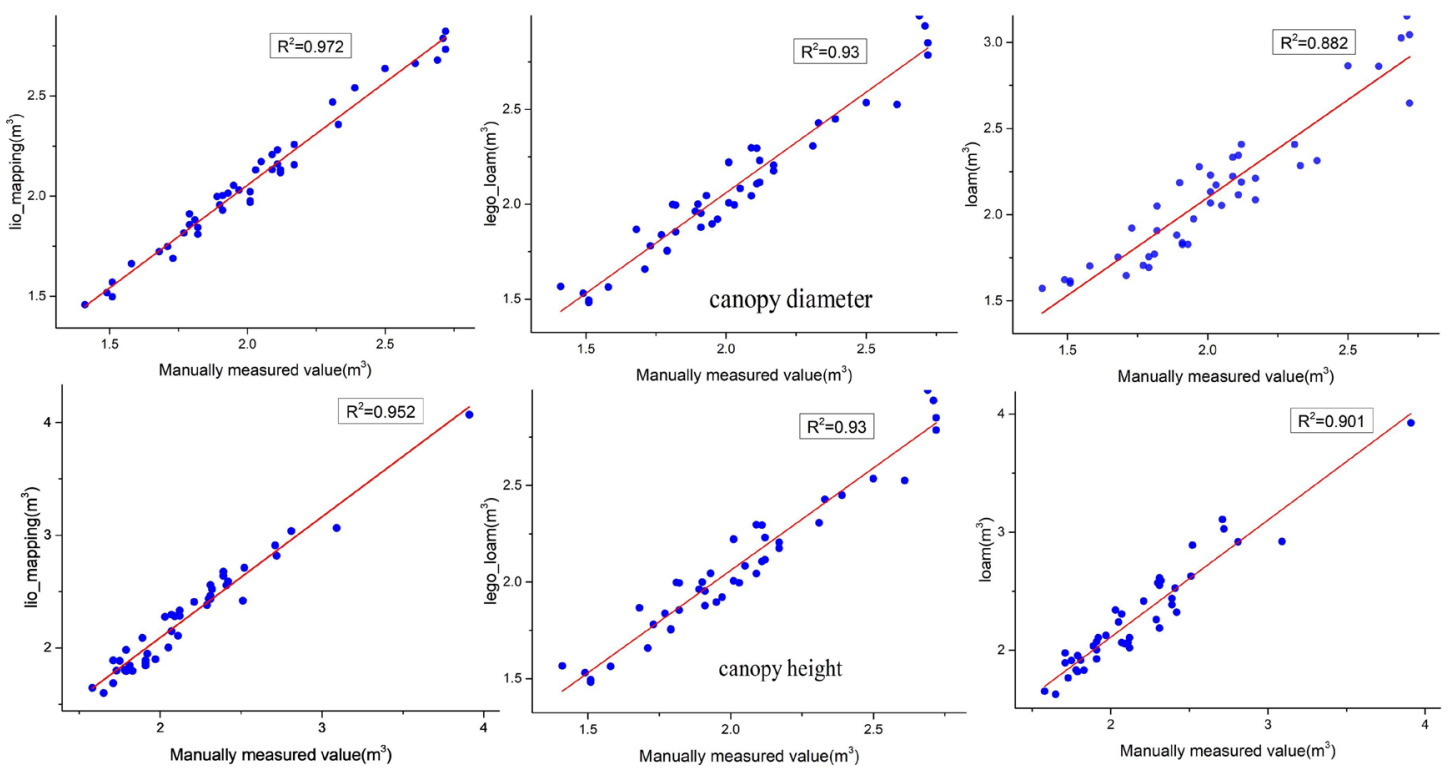

FIGURE 10. Correlation coefficient of canopy diameter and measurement under different algorithms

than the correlation coefficient between the crown height and the crown diameter, whose measurements were more accurate.

\section{CONCLUSION}

LiDAR SLAM technology has been applied to tree crown measurements, and a mobile visualization terminal was developed to enable it to be used for research on precision horticulture surveying, and mapping. To enhance the efficiency and robustness of the segmentation, humancomputer interactions, and European clustering were used to complete the tree segmentation of the measurement area. Statistical and voxel methods were used to extract the canopy morphology. Compared with the ground LiDAR, this method is advantageous in terms of both price and efficiency. To verify the reliability of the algorithm presented in this paper, comparative experiments with LOAM and LeGO-LOAM algorithms were conducted. The experiments showed that the method presented in this paper significantly improved the determination of the tree crown morphological parameters, when compared with LOAM and LeGOLOAM.

\section{ACKNOWLEDGEMENTS}

The authors wish to acknowledge the financial support from Primary Research \& Development Plan of Jiangsu Province (BE2017370).

\section{REFERENCES}

Agarwal, S., Mierle, K. \& Others. 2018. Ceres Solver. http:// ceres-solver.org/.

Davison, S., Donoghue, D.N.M. \& Galiatsatos, N. 2020. The effect of leaf-on and leaf-off forest canopy conditions on LiDAR derived estimations of forest structural diversity. International Journal of Applied Earth Observation and Geoinformation 92: 102160

Dong, Y., Li, Y., Li, Y., Li, P. \& L, Y. 2018. Tree crown outline point extracting and volume calculation based on improved convex hull algorithm. Engineering of Surveying and Mapping 27: 66-71.

Escolà, A., Martínez-Casasnovas, J.A., Rufat, J., Arnó, J., Arbonés, A., Sebé, F., Pascual, M., Gregorio, E. \& RosellPolo, J.R. 2016. Mobile terrestrial laser scanner applications in precision fruticulture/horticulture and tools to extract information from canopy point clouds. Precision Agriculture 18: 111-132.

Fan, Z., Feng, Z., Zheng, J., Fan, J., Yan, F. \& Qiu, Z. 2015. Tree crown volume calculation and prediction model establishment using cubic lattice method. Transactions of the Chinese Society for Agricultural Machinery 46(3): 321-327.

Hadas, E., Jozkow, G., Walicka, A. \& Borkowski, A. 2019. Apple orchard inventory with a LiDAR equipped unmanned aerial system. International Journal of Applied Earth Observation and Geoinformation 82: 101911.

Langning, H. \& Xiaoli, Z. 2019. A new method of equiangular sectorial voxelization of single-scan terrestrial laser scanning data and its applications in forest defoliation estimation. ISPRS Journal of Photogrammetry and Remote Sensing 151: 302-312. 
Li, Q., Zheng, J., Zhou, H., Zhang, H., Shu, Y. \& Xu, B. 2016. Online measurement of tree canopy volume using vehicleborne 2-D laser scanning. Transactions of the Chinese Society for Agricultural Machinery 47: 310-314.

Pedersen, K., Hulusic, V., Amelidis, P. \& Slattery, T. 2020. Spatialised audio in a custom-built openGL-based ear training virtual environment. IEEE Computer Graphics and Applications 4(5): 67-81.

Pfeiffer, A.S., Guevara, J., Cheein, F.A. \& Sanz, R. 2018. Mechatronic terrestrial LiDAR for canopy porosity and crown surface estimation. Computers and Electronics in Agriculture 146: 104-113.

Pierzchała, M., Giguère, P. \& Astrup, R. 2018. Mapping forests using an unmanned ground vehicle with $3 \mathrm{D}$ LiDAR and graph-SLAM. Computers and Electronics in Agriculture 145: 217-225.

Qian, X. \& Ye, C. 2014. NCC-RANSAC: A fast plane extraction method for 3-D range data segmentation. IEEE Trans 44(12): 2771-2783.

Qin, T., Li, P. \& Shen, S. 2018. Vins-mono: A robust and versatile monocular visual-inertial state estimator. IEEE Transactions on Robotics 34(4): 1004-1020.

Qin, C., Ye, H., Pranata, C.E., Han, J., Zhang, S. \& Liu, M. 2020. RLINS: A robocentric lidar-inertial state estimator for robust and efficient navigation. 2020 IEEE International Conference on Robotics and Automation (ICRA). pp. 8899-8906.

Rahul, K., Raheman, H. \& Paradkar, V. 2020. Design of a 4 DOF parallel robot arm and the firmware implementation on embedded system to transplant pot seedlings. Artificial Intelligence in Agriculture 4: 172-183.

Rusu, R.B. \& Cousins, S. 2011. Point cloud library (pcl). Proceedings of the 2011 IEEE International Conference on Robotics and Automation. pp. 1-4.

Segal, A., Haehnel, D. \& Thru, S. 2009. Generalized-ICP. Proceedings of Robotics: Science and Systems 2(4): 435.

Seka, D., Bonny, B.S., Yoboué, A.N., Sié, S.R. \& AdopoGourène, B.A. 2019. Identification of maize (Zea mays L.) progeny genotypes based on two probabilistic approaches: Logistic regression and naïve Bayes. Artificial Intelligence in Agriculture 1: 9-13.

Shan, T. \& Englot, B. 2018. LeGO-LOAM: Lightweight and ground optimized lidar odometry and mapping on variable terrain. IEEE/RSJ International Conference on Intelligent Robots and Systems. pp. 4758-4765.
Sun, G., Wang, X., Ding, Y., Lu, W. \& Sun, Y. 2019. Remote measurement of apple orchard canopy information using unmanned aerial vehicle photogrammetry. Agronomy 9(11): 744.

Underwood, J.P., Hung, C., Whelan, B. \& Sukkarieh, S. 2016. Mapping almond orchard canopy volume, flowers, fruit and yield using lidar and vision sensors. Computers and Electronics in Agriculture 130: 83-96.

Wang, J., Zhang, F., Gao, H. \& Lu, C. 2018. Extracting crown structure parameters of individual tree by using groundbased laser scanner. Transactions of the Chinese Society for Agricultural Machinery 49: 200-206.

Ye, H., Chen, Y. \& Liu, M. 2019. Tightly coupled 3D lidar inertial odometry and mapping. International Conference on Robotics and Automation (ICRA). pp. 3144-3150.

Zhang, J. \& Singh, S. 2016. Low-drift and real-time lidar odometry and mapping. Autonomous Robots 41: 401-416.

Zhang, J. \& Singh, S. 2014. LOAM: Lidar odometry and mapping in real-time. Proceedings of the Robotics: Science and Systems 2(9).

Zhou, S., Kang, F., Li, W., Kan, J., Zheng, Y. \& He, G. 2019. Extracting diameter at breast height with a handheld mobile LiDAR system in an outdoor environment. Sensors (Basel) 19(14): 3212.

Kai Wang, Wenhai Zhang \& Baohua Zhang

College of Engineering

Nanjing Agricultural University

Nanjing 210031

China

Jun Zhou*

Jiangsu Province Key Laboratory of Intelligent Agricultural Equipment

Nanjing 210031

China

*Corresponding author; email: zhoujun@njau.edu.cn

Received: 16 August 2020

Accepted: 7 March 2021 